\title{
Correction to: Applications of Artificial Intelligence in Business, Education and Healthcare
}

Allam Hamdan, Aboul Ella Hassanien, Reem Khamis, Bahaaeddin Alareeni, Anjum Razzaque, and Bahaa Awwad

\section{Correction to:}
A. Hamdan et al. (eds.), Applications of Artificial Intelligence in Business, Education and Healthcare, Studies in Computational Intelligence 993, https://doi.org/10.1007/978-3-030-72080-3

The original version of the book was inadvertently published with incorrect volume number, and this has been corrected as 993. This has now been amended throughout the book to the correct volume number. 